\title{
Post-mortem 1.5T MR quantification of regular anatomical brain structures
}

\author{
Wolf-Dieter Zech ${ }^{1,3}$ • Anna-Lena Hottinger ${ }^{1} \cdot$ Nicole Schwendener $^{1}$ • \\ Frederick Schuster $^{1,2} \cdot$ Anders Persson $^{3} \cdot$ Marcel J. Warntjes $^{3} \cdot$ Christian Jackowski $^{1}$
}

Received: 17 November 2015 / Accepted: 13 January 2016 /Published online: 12 February 2016

(C) Springer-Verlag Berlin Heidelberg 2016

\begin{abstract}
Recently, post-mortem MR quantification has been introduced to the field of post-mortem magnetic resonance imaging. By usage of a particular MR quantification sequence, $\mathrm{T} 1$ and $\mathrm{T} 2$ relaxation times and proton density (PD) of tissues and organs can be quantified simultaneously. The aim of the present basic research study was to assess the quantitative T1, T2, and PD values of regular anatomical brain structures for a $1.5 \mathrm{~T}$ application and to correlate the assessed values with corpse temperatures. In a prospective study, 30 forensic cases were MR-scanned with a quantification sequence prior to autopsy. Body temperature was assessed during MR scans. In synthetically calculated T1, T2, and PDweighted images, quantitative T1, T2 (both in ms) and PD (in \%) values of anatomical structures of cerebrum (Group 1: frontal gray matter, frontal white matter, thalamus, internal capsule, caudate nucleus, putamen, and globus pallidus) and brainstem/cerebellum (Group 2: cerebral crus, substantia nigra, red nucleus, pons, cerebellar hemisphere, and superior cerebellar peduncle) were assessed. The investigated brain structures of cerebrum and brainstem/cerebellum could be characterized and differentiated based on a combination of their quantitative T1, T2, and PD values. MANOVA testing verified significant differences between the investigated
\end{abstract}

Wolf-Dieter Zech

Wolf-Dieter.Zech@irm.unibe.ch

1 Institute of Forensic Medicine, University of Bern, Buehlstrasse 20, 3012 Bern, Switzerland

2 Department of Diagnostic, Interventional and Pediatric Radiology, University of Bern, Inselspital, Freiburgstrasse 10, Bern CH-3010, Switzerland

3 Center for Medical Image Science and Visualization (CMIV), University of Linköping, 58185 Linköping, Sweden anatomical brain structures among each other in Group 1 and Group 2 based on their quantitative values. Temperature dependence was observed mainly for T1 values, which were slightly increasing with rising temperature in the investigated brain structures in both groups. The results provide a base for future computer-aided diagnosis of brain pathologies and lesions in post-mortem magnetic resonance imaging.

Keywords Brain · MRquantification · Post-mortem magnetic resonance imaging $(\mathrm{PMMR}) \cdot$ Forensic $\cdot$ Neuroimaging

\section{Introduction}

Recently, post-mortem MR quantification has been introduced to the field of post-mortem magnetic resonance imaging (PMMR) [1-5]. By usage of a particular MR quantification sequence, $\mathrm{T} 1$ and $\mathrm{T} 2$ relaxation times (in milliseconds) and Proton density (PD in \%, where $100 \%$ is equivalent to pure water) of tissues can be quantified simultaneously [6-9]. Regular tissues and pathologies can be described by quantitative measurements within synthetically calculated MR images. By a combination of T1, T2, and PD values that are not vendor specific, each tissue can be characterized and discerned from lesions or pathologies $[3,4]$. The quantification approach provides a base for computer-aided diagnosis (CAD) of lesions and forensic relevant findings $[10,11]$. In order to implement CAD in post-mortem forensic MR imaging, databases of quantitative values of regular tissues, pathologic tissue and tissue lesions have to be established for relevant organs and tissues. Since quantitative values of various tissues, especially T1 values have shown to be temperature dependent, there is a need for temperature correction of quantitative values [2-4, 12]. So far, simultaneous quantification of $T 1, T 2$, and $P D$ values in a post-mortem MR quantification setting has only 
been applied to thoraco-abdominal organs, tissues and body fluids. It could be demonstrated that based on the combination of quantitative T1, T2, and PD values, abdominal organs as well as body fluids could be differentiated from each other [3, 5]. Moreover, ischemic and hemorrhagic myocardial lesions could be differentiated from normal myocardium based on the combination of their quantitative T1, T2, and PD values [4]. In forensic neuroimaging, the quantification approach bears great potential since it might be feasible for automated detection of ischemia, hemorrhages, inflammation and tumors $[1,4$, $9,13]$. In order to use the quantification approach for assessment of brain pathologies and lesions, the quantitative T1, T2, and PD values of the regular anatomical brain structures have to be assessed first. Knowledge of the quantitative values of the regular anatomical brain structures provides a base for discrimination between healthy and altered brain tissues. The aim of the present study was to assess the quantitative $\mathrm{T} 1, \mathrm{~T} 2$, and PD values of regular post-mortem anatomical structures of the cerebrum and brainstem for a $1.5 \mathrm{~T}$ application and to correlate the assessed values with corpse temperatures.

\section{Methods}

\section{Study population}

In 30 forensic cases (13 female, 17 male), a quantification sequence was added to a post-mortem Neuro MR examination in a prospective study. The age at death ranged from 22 to 82 years (mean age 50.9 years, SD 16.4). The post-mortem interval (PMI: time between death and MR examination) ranged from 1 to 3 days. Cases were chosen selectively according to the following inclusion and exclusion criteria: only brains which appeared unremarkable at autopsy were included (unremarkable anatomical brain structures, no severe signs of edema (flattening of gyri, narrowing of sulci, expansion of white matter with reduction of the ventricular system, or extension of cones at basal part of cerebellum), and no brain injuries or brain pathologies). Exclusion criteria were counter wise to the latter and age less than 18 years. Corpses with relevant external signs of putrefaction (brown- to green-colored skin veins, ablation of external skin layers and vesicle formations of the skin) were also excluded. Table 1 gives information on all cases including age, gender, manner/ cause of death, PMI and body core temperature at MR examination. Due to logistic infrastructure no further information about the included corpses was available. According to autopsy reports estimated time of death was given in time ranges (maximal and minimal time of death). The mean of minimal and maximal time of death was used for determining the PMI.

\section{PMCT and magnetic resonance imaging}

All corpses underwent post-mortem computed tomography (PMCT) scanning prior to MR examination. Corpses with relevant putrefaction gas formations in $\mathrm{CT}$ images were also excluded from this study. Gas formations in PMCT were evaluated according to the Radiological alteration index (RAI) from Egger et al. [14]. Corpses with a RAI $>50$ were considered to exhibit relevant putrefaction. Usage of the acquired MRI data was approved by the local ethics committee. For MR examination (Siemens Magnetom Symphony Tim $1.5 \mathrm{~T}$ ), corpses were wrapped in an artifact-free body bag. Subjects were examined in the supine position. The MR examination time was $45 \mathrm{~min}$, of which the quantification sequence lasted $15 \mathrm{~min}$. The MRI quantification sequence was a multi-slice turbo spin echo (TSE) sequence, where each acquisition was performed with eight different echo-times (TE) at multiples of $13.5 \mathrm{~ms}$ [13.5-108 ms]. Four different saturation delay times were acquired at 100, 400, 1100, and $2400 \mathrm{~ms}$, using a repetition time (TR) of $2500 \mathrm{~ms}$. In this setup, 32 different images were acquired per slice, with different effects of $\mathrm{T} 1$ and $\mathrm{T} 2$ relaxation. $\mathrm{T} 1$ and $\mathrm{T} 2$ relaxation, as well as proton density (PD), were retrieved by a commercially available post-processing tool (SyMRI Autopsy, SyntheticMR, Linköping, Sweden) [15]. The same software provided synthetic T1-weighted (T1w), T2-weighted (T2w), and PD-weighted (PDw) images for visual support. Twenty axial brain slices of $5 \mathrm{~mm}$ thickness were acquired with a gap of $0.3 \mathrm{~mm}$. In each case, additional conventional T1 and T2 weighted axial brain images with a slice thickness of $5 \mathrm{~mm}$ and a gap of $0.3 \mathrm{~mm}$ were acquired with the following scan parameters: T1w: TSE $(\mathrm{TE}=9 \mathrm{~ms}, \mathrm{TR}=500 \mathrm{~ms}) ; \mathrm{T} 2 \mathrm{w}$ : TSE $(\mathrm{TE}=92 \mathrm{~ms}, \mathrm{TR}=5000 \mathrm{~ms}$ ). During the MRI scans, the corpse temperatures were assessed in real time with MRcompatible temperature probes that were placed into the esophagus before the MR examination to assess body core temperature at the time of data acquisition. The body core temperatures of corpses ranged from 8 to $35.1{ }^{\circ} \mathrm{C}$ (mean temperature $20.1{ }^{\circ} \mathrm{C}, \mathrm{SD} 6.6$ ) (Table 1).

\section{Forensic autopsy and brain dissection}

Forensic autopsies were ordered by the local authorities. Autopsies were carried out by board certified forensic pathologists immediately after MR examinations. After dissection of the scalp the skull-cap was sawn circumferentially with an oscillating saw. The skull-cap was pried loose by shifting a T-shaped chisel in the saw cut. Afterwards the brain was cut into two halves using the Flechsig technique and removed from the skull. Both brain halves, including the cerebellum and brain stem were removed from the opened skull. Further dissection of the brain was performed by parallel slicing of the two brains halves. In each case brain tissue samples were 
Table 1 Age, sex, manner and cause of death, PMI and body temperature (assessed during MR scan) in the quantified 30 forensic cases

\begin{tabular}{|c|c|c|c|c|c|}
\hline Case no. & Sex & Age (y) & Manner and cause of death & PMI (h) & Temperature $\left({ }^{\circ} \mathrm{C}\right)$ \\
\hline 1 & $\mathrm{~F}$ & 63 & Natural-Pulmonary Embolism & 12 & 31.2 \\
\hline 2 & $\mathrm{~F}$ & 40 & Natural—Cardiac Arrest & 22 & 19 \\
\hline 3 & $\mathrm{~F}$ & 46 & Natural - Cardiac Arrest & 10 & 20.5 \\
\hline 4 & M & 53 & Natural_-Pericardial Tamponade & 68 & 15.7 \\
\hline 5 & $\mathrm{~F}$ & 58 & Natural-Myocardial Infarction & 24 & 26 \\
\hline 6 & M & 47 & Natural—Pulmonary Embolism & 72 & 16.6 \\
\hline 7 & M & 57 & Natural - Cardiac Arrest & 30 & 28.4 \\
\hline 8 & $\mathrm{~F}$ & 69 & Natural - Cardiac Arrest & 24 & 21.5 \\
\hline 9 & M & 43 & Natural - Cardiac Arrest & 44 & 10.8 \\
\hline 10 & $\mathrm{~F}$ & 49 & Accident—Ketoacidosis & 48 & 18.7 \\
\hline 11 & M & 56 & Accident-Trauma to Torso & 12 & 24.5 \\
\hline 12 & $\mathrm{~F}$ & 76 & Natural_Pneumonia & 26 & 19.3 \\
\hline 13 & $\mathrm{~F}$ & 50 & Natural-Endocarditis & 60 & 22.1 \\
\hline 14 & M & 30 & Accident-Intoxication & 6 & 35.1 \\
\hline 15 & M & 23 & Accident-Thorax Trauma & 24 & 17.2 \\
\hline 16 & M & 82 & Accident—Fat Embolism & 48 & 18.6 \\
\hline 17 & M & 48 & Accident-Thorax Trauma & 62 & 16 \\
\hline 18 & M & 48 & Natural - Cardiac Arrest & 36 & 10.8 \\
\hline 19 & $\mathrm{~F}$ & 37 & Accident-Aspiration & 58 & 18.3 \\
\hline 20 & M & 65 & Suicide - Intoxication & 24 & 27.7 \\
\hline 21 & M & 59 & Suicide - Intoxication & 40 & 21.9 \\
\hline 22 & $\mathrm{~F}$ & 80 & Accident-Abdominal Trauma & 18 & 31.7 \\
\hline 23 & $\mathrm{~F}$ & 46 & Natural-Undetermined & 12 & 26.7 \\
\hline 24 & M & 81 & Accident—Fat Embolism & 36 & 9.5 \\
\hline 25 & M & 47 & Natural_-Undetermined & 24 & 16.1 \\
\hline 26 & M & 22 & Accident-Intoxication & 48 & 18.3 \\
\hline 27 & $\mathrm{~F}$ & 24 & Accident—Abdominal Trauma & 40 & 18.8 \\
\hline 28 & $\mathrm{~F}$ & 51 & Suicide - Thorax Trauma & 72 & 8 \\
\hline 29 & M & 32 & Accident-Intoxication & 65 & 17.1 \\
\hline 30 & M & 45 & Natural_-Undetermined & 26 & 18.3 \\
\hline
\end{tabular}

collected (frontal cortex, hippocampus, cerebellum, and pons) for histologic examinations (stain Hematoxylin and Eosin (H\&E)). Histologic evaluation was conducted by board certified forensic pathologists who were blinded for the MRI findings. Histology samples were obtained and investigated as part of the routine post-mortem examination, which was ordered by the local authorities. The samples were not taken for the purpose of the study and are taken in any case independently from any study. The post-mortem imaging findings obtained in the study were compared to the already existing histology reports as obtained in routine forensic case work.

\section{Acquisition of quantitative values of regular anatomical brain structures}

In synthetically calculated axial brain T1, T2, and PD-weighted images, quantitative T1, T2 (both in ms), and PD (in \%) values of anatomical brain structures were assessed. The anatomical brain structures were assigned to two different groups. Group 1 included the following structures of the cerebrum: frontal gray matter, frontal white matter, thalamus, internal capsule, caudate nucleus (caput), putamen, and globus pallidus. Group 2 included the following structures of the brain stem and cerebellum: cerebral crus, substantia nigra, red nucleus, pons, cerebellar hemisphere, and superior cerebellar peduncle. A total of six independent (three on each hemisphere) and non-overlapping square regions of interest (ROIs) were placed in the defined structures in two adjacent slices (Fig. 1). The size of the ROIs depended on the size of the measured brain structure, but was at least $0.4 \mathrm{~cm}$ in each dimension. All measurements were conducted by one observer.

\section{Temperature correction of quantitative values}

Microsoft Excel ${ }$ was used to generate linear equations from the T1/temperature, T2/temperature, and $\mathrm{PD} /$ temperature 
Fig. 1 Example of quantitative measurement conducted in a synthetically calculated PDw MR image. In this case quantitative values of the thalamus were obtained by placing a ROI (green arrow) into the thalamic region. $\mathrm{T} 1$ and $\mathrm{T} 2$ values (both in $\mathrm{ms}$ ) and PD (as \%) of the measured thalamic region are given in the upper right corner (red frame) of the image

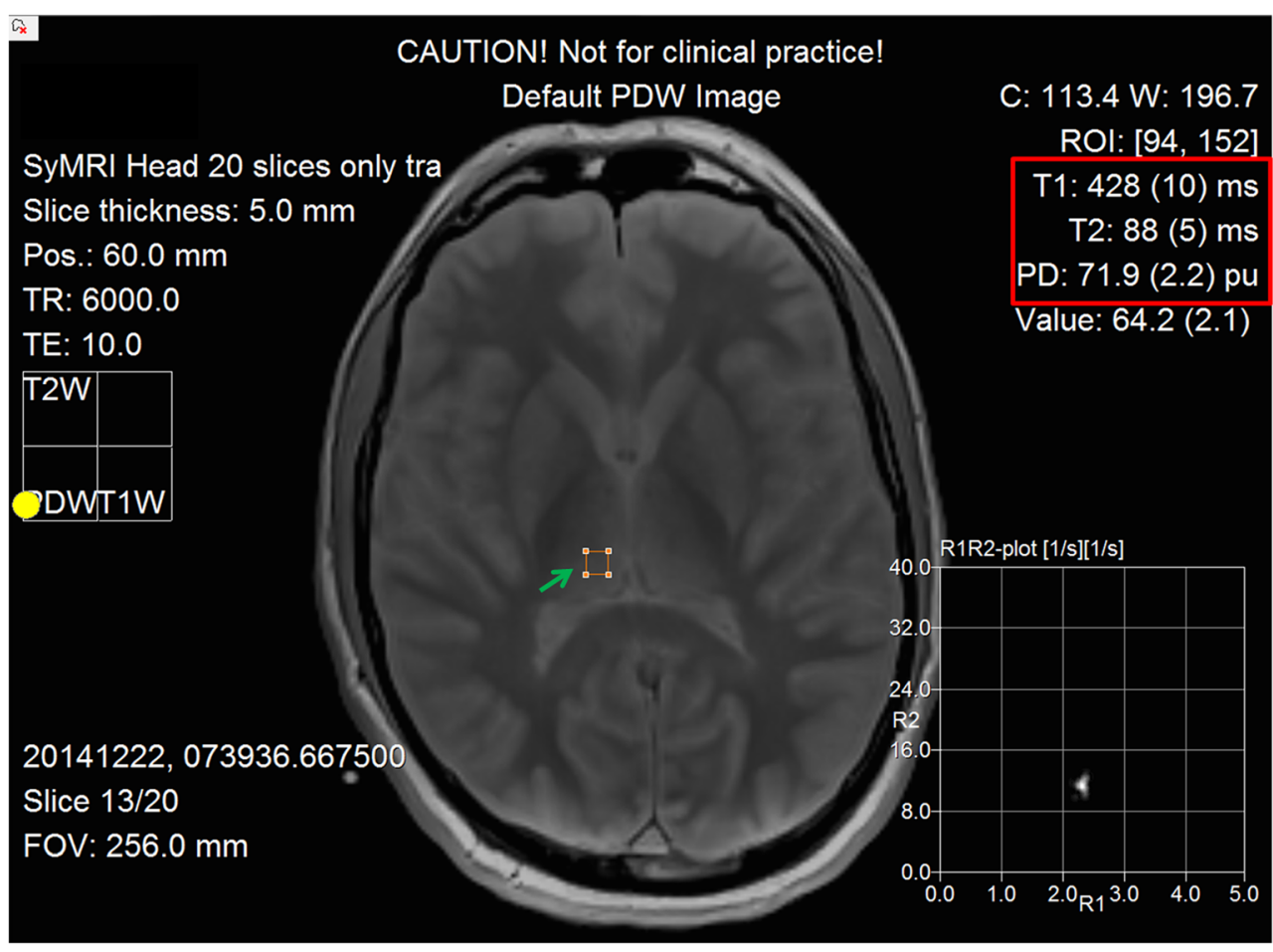

relationships for each of the investigated anatomical brain structures. Those equations were used to correct the values of T1, T2, and PD to $37^{\circ} \mathrm{C}$ : the according corpse temperatures were subtracted from $37^{\circ} \mathrm{C}$ ( $\Delta$ temperatures). $\Delta$ temperatures were applied in the equations generated from the quantitative values/temperature relation graphs to gain $\Delta \mathrm{T} 1, \Delta \mathrm{T} 2$, and $\Delta$ PD. Those $\Delta$ values were summated to the uncorrected T1, $\mathrm{T} 2$, and $\mathrm{PD}$ values to gain the temperature corrected values $[3]$.

\section{Visualization of quantitative values}

To visualize clustering and separation of the quantitative values of brain structures in Group 1 and Group 2 the mean quantitative T1, T2 and PD values were plotted in a 3D Plot using the ThreeDify Excel Grapher add-in for Microsoft Excel®.

\section{Statistical analyses}

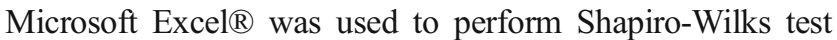
to evaluate normal distribution of quantitative values. Since all quantitative values showed normal distribution, a series of MANOVAs (multiple analysis of variance) was performed to evaluate significant differentiability of quantitative T1, T2, and PD values between the investigated brain structures among each other in Group 1 and Group 2. In the MANOVA tests $p$ values $<0.05$ were considered statistically significant. Regression analysis for correlation of non-temperature-corrected quantitative T1,
T2, and PD values of all assessed anatomical brain structures with post-mortem intervals, and the age and sex of the study population were analyzed using the Pearson product moment correlation. $p$ values $<0.05$ were assumed to be statistically significant.

Using the MATLAB ${ }^{\circledR}$ software the receiver operator characteristic (ROC) curve approach was applied to give accuracy of discrimination between the tested anatomical structures in both groups. Accuracy was measured by the area under the ROC curve (AUC) with the traditional academic point system: $0.90-1=$ excellent; $0.80-0.90=$ good; $0.70-0.80=$ fair; $0.60-$ $0.70=$ poor; and $0.50-0.60=$ fail.

\section{Results}

\section{Temperature dependence of quantitative values}

The relations of quantitative $\mathrm{T} 1, \mathrm{~T} 2$, and $\mathrm{PD}$ values and temperature in the investigated brain structures of Group 1 and Group 2 were expressed by linear equations. Table 2 gives the linear equations generated for all assessed brain structures. Temperature dependence was observed mainly for T1 values, which were slightly increasing with rising temperature in the investigated brain structures in both groups (Fig. 2). Individual variation of the temperature/quantitative T1, T2, and PD value relations was observed in all investigated brain structures in both groups (Table 2). 
Table 2 Linear equations for regular anatomical brain structures of cerebrum (Group 1) and brainstem/cerebellum (Group 2) generated from the relation of T1, T2, and PD to body core temperature at the time of scanning as obtained from 30 forensic cases

\begin{tabular}{llll}
\hline & T1/temperature & T2/temperature & PD/temperature \\
\hline Group 1 & & & \\
$\quad$ Gray matter & $y=0.97 x+472.51$ & $y=0.66 x+104.10$ & $y=0.14 x+80.39$ \\
White matter & $y=1.99 x+404.84$ & $y=0.11 x+100.51$ & $y=0.007 x+61.96$ \\
Caudate nucleus & $y=1.21 x+443.45$ & $y=0.12 x+84.83$ & $y=-0.62 x+91.79$ \\
Putamen & $y=0.83 x+419.26$ & $y=0.29 x+72.01$ & $y=-0.13 x+76.08$ \\
Globus pallidus & $y=0.51 x+407.07$ & $y=-0.01 x+76.62$ & $y=0.03 x+66.54$ \\
Thalamus & $y=1.95 x+399.20$ & $y=0.55 x+78.13$ & $y=0.19 x+66.97$ \\
Internal capsule & $y=1.48 x+398.93$ & $y=0.39 x+105.79$ & $y=0.12 x+51.06$ \\
Group 2 & & & \\
Cerebral crus & $y=1.01 x+427.75$ & $y=0.09 x+109.62$ & $y=0.08 x+54.21$ \\
Substantia nigra & $y=0.29 x+442.55$ & $y=0.24 x+82.02$ & $y=0.08 x+69.73$ \\
Red nucleus & $y=1.24 x+433.29$ & $y=-0.40 x+115.32$ & $y=0.09 x+56.68$ \\
Pons & $y=1.30 x+405.28$ & $y=0.43 x+89.69$ & $y=0.13 x+62.25$ \\
Superior cerebellar peduncle & $y=1.26 x+417.24$ & $y=0.10 x+111.42$ & $y=0.08 x+63.72$ \\
Cerebellum & $y=2.41 x+414.13$ & $y=0.62 x+83.00$ & $y=0.13 x+69.09$ \\
\hline
\end{tabular}

$T 1 \mathrm{~T} 1$ relaxation time in milliseconds, $T 2 \mathrm{~T} 2$ relaxation time in milliseconds, $T$ body core temperature in ${ }^{\circ} \mathrm{C}, P D$ proton density in $\%$ related to pure water $(100 \%)$

\section{Histologic examinations}

Histologic examinations revealed signs of slight cerebral edema (slight distension of perivascular and pericellular spaces, slight rarefaction of subpial spaces, and slight vacuolar appearance of gray matter neuropil) in all investigated brains. Apart from that no remarkable findings were present in the investigated brain slices.
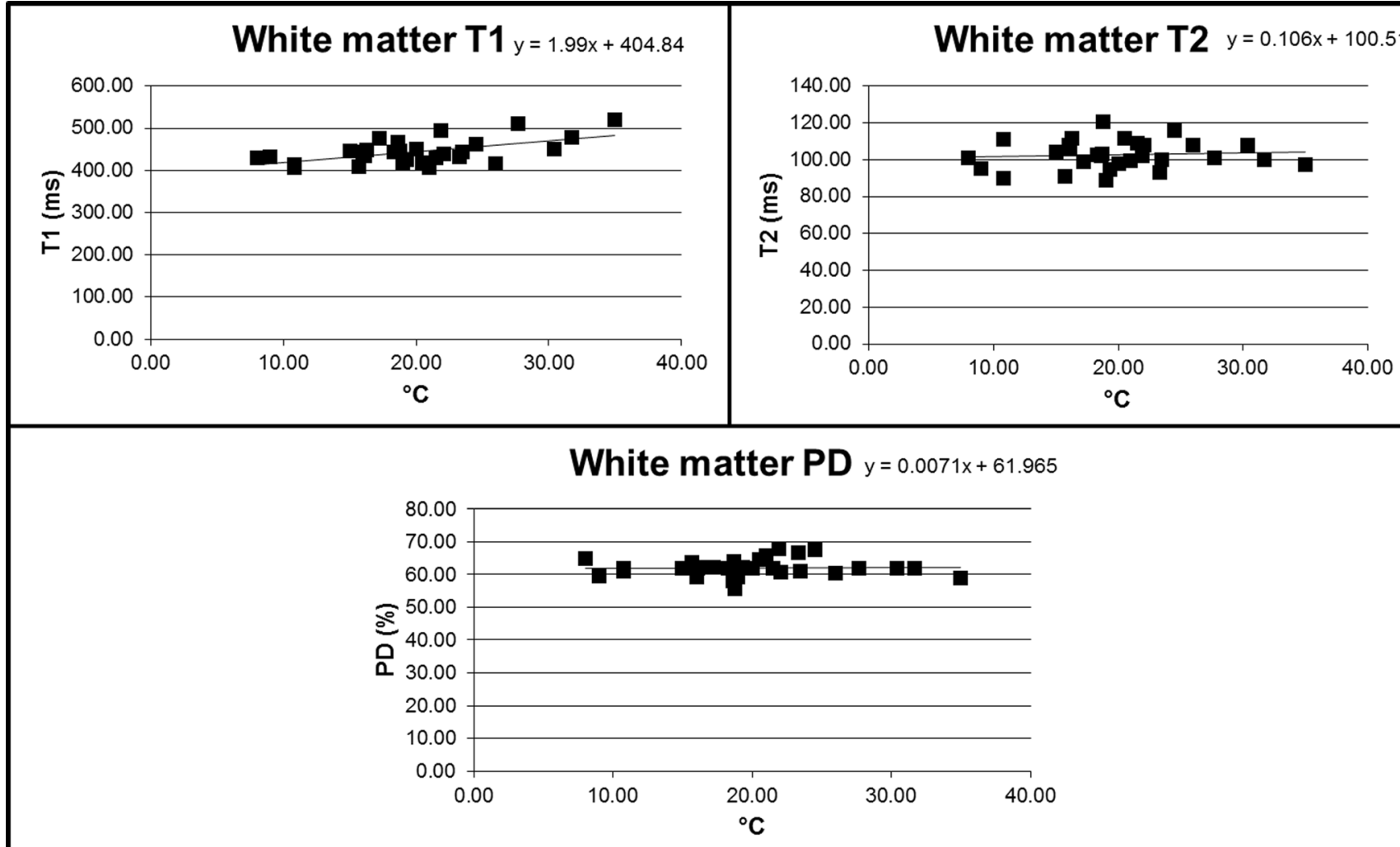

Fig. 2 Example of temperature dependence of quantitative values: plots of $\mathrm{T} 1$ and $\mathrm{T} 2$ relaxation times and PD of frontal white matter versus body core temperature at the time of scanning. There is temperature dependence mainly for the $\mathrm{T} 1$ values. It was assumed that the quantitative $\mathrm{T} 1$ values correlate linearly with rising temperature. $\mathrm{T} 2$ and $\mathrm{PD}$ values are only slightly influenced by temperature 
Table 3 Mean quantification values (T1 and T2 in ms; PD in \%; obtained from six measurements) and standard deviations (SD) of the investigated anatomical brain structures in cerebrum (Group 1) and brainstem/cerebellum (Group 2) in 30 forensic cases

\begin{tabular}{|c|c|c|c|c|c|c|}
\hline & \multicolumn{2}{|l|}{$\mathrm{T} 1$ (SD) } & \multicolumn{2}{|l|}{$\mathrm{T} 2$ (SD) } & \multicolumn{2}{|l|}{$\mathrm{PD}(\mathrm{SD})$} \\
\hline & Uncorrected & Corrected to $37^{\circ} \mathrm{C}$ & Uncorrected & Corrected to $37^{\circ} \mathrm{C}$ & Uncorrected & Corrected to $37^{\circ} \mathrm{C}$ \\
\hline \multicolumn{7}{|l|}{ Group 1} \\
\hline Gray matter & $493.08(24.13)$ & $510.03(23.58)$ & $116.73(11.71)$ & $128.26(10.91)$ & $83.17(2.23)$ & $85.61(2.05)$ \\
\hline White matter & $443.4(29.07)$ & $478.18(26.22)$ & $113.95(16.54)$ & $130.38(15.48)$ & $62.89(3.56)$ & $66.73(3.42)$ \\
\hline Caudate nucleus & $439.37(25.21)$ & $435.7(25.17)$ & $87.19(11.86)$ & $89.29(11.84)$ & $79.69(8.12)$ & $78.85(7.15)$ \\
\hline Putamen & $435.43(24.50)$ & $449.93(23.95)$ & $77.75(10.76)$ & $82.81(10.6)$ & $73.63(3.29)$ & $71.35(3.19)$ \\
\hline Globus pallidus & $416.99(28.20)$ & $425.9(28.02)$ & $76.54(13.86)$ & $76.37(13.08)$ & $67.21(2.58)$ & $67.91(2.57)$ \\
\hline Thalamus & $436.24(22.26)$ & $470.32(21.13)$ & $88.8(8.55)$ & $98.41(7.85)$ & $70.59(5.10)$ & $73.91(4.95)$ \\
\hline Internal capsule & $427.06(20.51)$ & $452.93(18.14)$ & $113.27(9.70)$ & $120.09(9.36)$ & $53.35(9.56)$ & $55.44(1.89)$ \\
\hline \multicolumn{7}{|l|}{ Group 2} \\
\hline Cerebral crus & $427.73(19.84)$ & $427.71(18.12)$ & $111.48(11.58)$ & 113.05 (11.57) & $55.89(5.59)$ & $57.29(5.47)$ \\
\hline Substantia nigra & $448.18(20.48)$ & $453.25(20.26)$ & $86.62(10.40)$ & $90.82(10.29)$ & $71.2(5.88)$ & $72.6(5.86)$ \\
\hline Red nucleus & $428.64(29.39)$ & $424.45(29.26)$ & $107.47(16.38)$ & $100.47(9.63)$ & $58.35(4.51)$ & $59.93(4.48)$ \\
\hline Pons & $430.93(19.05)$ & $453.65(17.35)$ & $98.14(10)$ & $105.66(9.63)$ & $64.6(3.74)$ & $66.87(3.64)$ \\
\hline Superior cerebellar peduncle & $441.89(17.7)$ & $463.91(15.87)$ & $113.4(11.82)$ & $115.15(11.3)$ & $65.32(4.52)$ & $66.71(4.49)$ \\
\hline Cerebellum & $460.11(27.67)$ & $502.23(22.92)$ & $95.11(9.16)$ & $105.95(8.3)$ & $71.64(2.56)$ & $73.91(2.43)$ \\
\hline
\end{tabular}

Data are presented with and without temperature correction to $37^{\circ} \mathrm{C}$

\section{Visualization and statistical differentiability of quantitative values}

Table 3 shows the assessed mean T1, T2 and PD quantification values and standard deviations of the investigated anatomical brain structures in both groups. Figure 3 depicts $3 \mathrm{D}$ plots of the temperature corrected quantitative T1, T2, and PD values of the investigated brain structures in Group 1 and Group 2. MANOVA testing verified significant differences between the investigated anatomical brain structures among each other in Group 1 and Group 2 based on their quantitative values (Table 4). Based on the ROC curve approach accuracy of discrimination between anatomical structures was excellent or good in all tested pairs of both groups (Table 4).

\section{Influence of age, sex and PMI on quantitative values}

Regression analysis revealed no significant correlation of the quantitative values for $\mathrm{T} 1, \mathrm{~T} 2$, and $\mathrm{PD}$ of the investigated brain structures compared with age and sex of the deceased, as well as the post-mortem interval (all $p$ values $>0.05$ ).

\section{Discussion}

In the present study, the quantitative T1, T2, and PD values of various relevant regular post-mortem anatomical structures of the cerebrum, brainstem, and cerebellum could be quantified. In both the cerebrum and brainstem/cerebellum, the assessed anatomical structures could be differentiated based on a combination of their quantitative T1, T2, and PD values. In the next step relevant brain pathologies and lesions need to be quantified and compared to the assessed regular anatomical brain structures. Based on previous work, it can be expected that the quantitative values of certain pathologies and lesions such as tumors, acute and chronic infarction, hemorrhages, and inflammation are differentiable to the quantitative values of the regular anatomical structures. Moreover, it may also be possible to estimate the age of hemorrhages and blood clots $[1,4,5,9,16,17]$. Once the quantitative values of regular anatomical brain structures, lesions, and pathologies are known they can be implemented in quantification databases. Such databases can be used to develop computer-aided diagnosis (CAD) software based on T1, T2, and PD quantification values. In a final step, such CAD-software could be implemented in a regular radiological workstation to aid the forensic radiologist in routine forensic casework [18-22]. Besides the possibility of the automated detection of pathologies, the quantitative MR datasets also offer the theoretic technical possibilities of MR image plan reconstruction and volume rendering on radiological workstations $[1,18]$. The final goal of the publication's host institute is to develop databases which contain the quantitative values of all relevant organs and tissues to establish a forensic CAD-workstation for whole body MR scans. Since the brain is a forensic, highly relevant organ assessment of quantitative brain data is inevitable for that purpose [23-29]. 


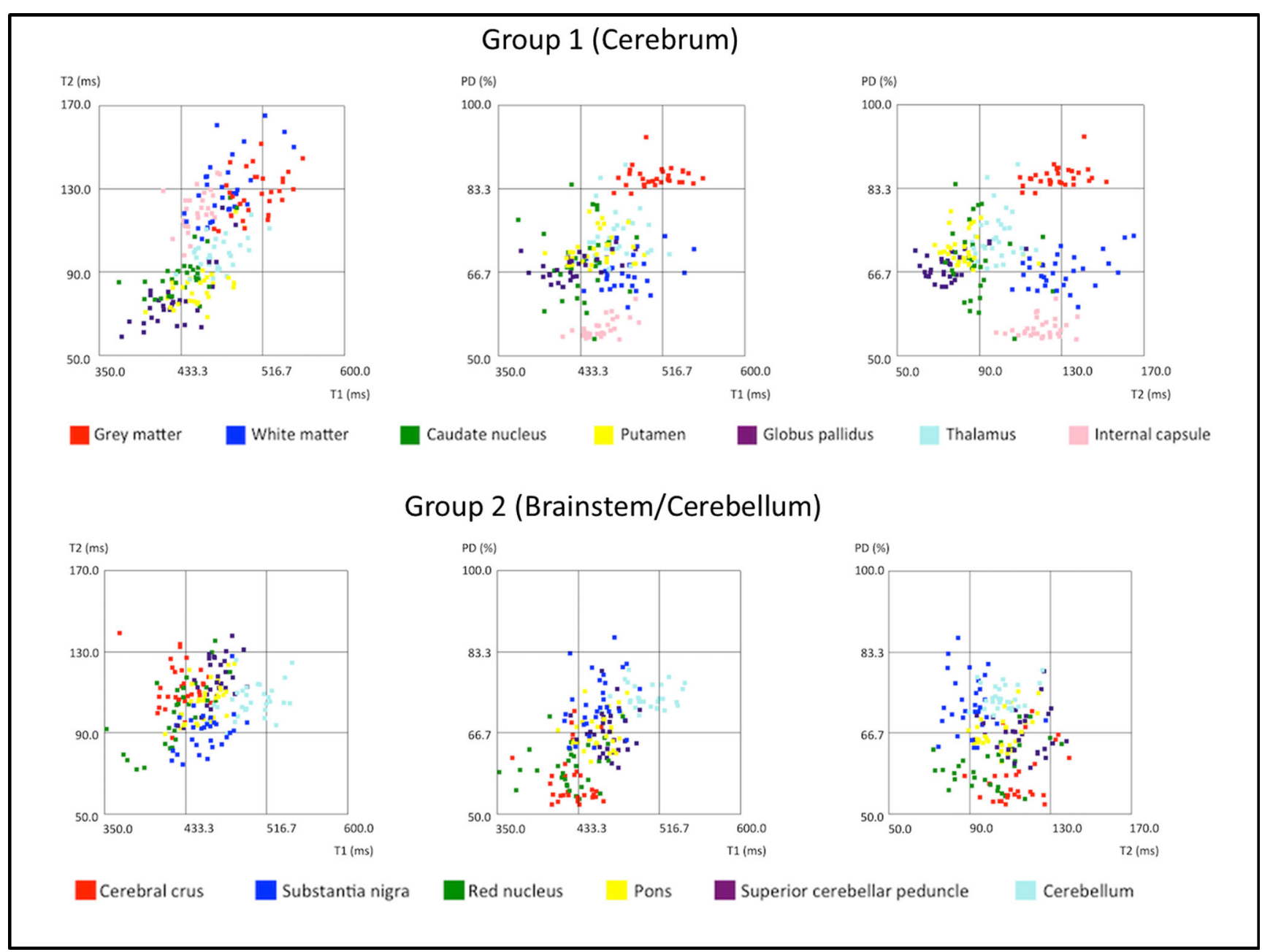

Fig. 3 3D plots of quantitative values (corrected for a temperature of $37^{\circ} \mathrm{C}$ ) of regular anatomical structures of the cerebrum (Group 1) and brainstem/cerebellum (Group 2). Three defined views (T2/T1 view, PD/

In accordance to previous studies a temperature dependence mainly of the $\mathrm{T} 1$ values could be assessed in all investigated structures in cerebrum and brainstem/cerebellum $[2,3$, $30,31]$. This reinforces the need to perform temperature corrections on quantitative values. If quantification values of corpses with different temperatures are to be compared, it is necessary to use temperature corrected values. The present study provides equations which can be used for temperature corrections. CAD-software based on quantitative values would have to consider corpse temperature and could use such equations for temperature correction of quantitative values.

All investigated brains showed histological signs of slight cerebral edema although the brains appeared unremarkable at gross examination at autopsy. It may be concluded that at least slight histologic signs of brain edema are a common finding in each post-mortem case and need to be taken into account for MR quantification. The reason for the appearance of slight signs of cerebral edema in causes of death not directly involving
$\mathrm{T} 1$ view, and $\mathrm{PD} / \mathrm{T} 2$ view) are shown for each group. In both groups, the best visual differentiation of the clusters from each other can be observed in the $\mathrm{PD} / \mathrm{T} 2$ views

the brain may be hypoxia during agony. Agony can last at least several minutes which may be enough time to cause hypoxia in the brain due to depraved circulation and blood oxygenation [32-35]. Slightly elevated water content in the brain due to edema may also influence quantitative values. Along with changes of $\mathrm{pH}$ value and tissue temperature, this may explain why the postmortem quantification values of brain tissue, especially the T1 values, differ from the known in vivo values of $\mathrm{T} 1$ and $\mathrm{T} 2$ relaxation times [2]. In some of the investigated cases, the cause of death was intoxication which is frequently, but not obligatory, accompanied by severe brain edema. However, these cases showed no severe edema at gross examination and in histological examinations and could, therefore, be included in this study. Case numbers for the present study were relatively small because gross signs of brain edema are a frequent finding at autopsy. Hence, many of our forensic autopsy cases did not fulfill the inclusion criteria of this study. 
Table 4 Results of MANOVA testing of T1 (ms), T2 (ms), and PD (\%) values (corrected to a temperature of $37^{\circ} \mathrm{C}$ ) in cerebrum (Group 1) and brainstem/cerebellum (Group 2) in 30 forensic cases. A significant difference between all tested anatomical structures can be determined in each group. The table also gives the area under the ROC curve (ROC/AUC) for the tested anatomical structures (corrected to $37^{\circ} \mathrm{C}$ ). Accuracy of discrimination between anatomical structures was excellent or good in all tested pairs of both groups (column at right side)

\begin{tabular}{|c|c|c|c|c|c|}
\hline MANOVA for $\mathrm{T} 1, \mathrm{~T} 2$, and PD values & Stat value & Test value & $p$ value & Significance & ROC-AUC/accuracy \\
\hline \multicolumn{6}{|l|}{ Group 1} \\
\hline Gray matter-white matter & 0.94 & 281.00 & $<0.05$ & Yes & 0.98/excellent \\
\hline Gray matter-caudate nucleus & 0.87 & 119.67 & $<0.05$ & Yes & 1/excellent \\
\hline Gray matter-putamen & 0.92 & 207.14 & $<0.05$ & Yes & 1/excellent \\
\hline Gray matter-globus pallidus & 0.94 & 295.03 & $<0.05$ & Yes & 1/excellent \\
\hline Gray matter-thalamus & 0.81 & 80.50 & $<0.05$ & Yes & 0.94/excellent \\
\hline Gray matter-internal capsule & 0.98 & 1193.66 & $<0.05$ & Yes & 0.96/excellent \\
\hline White matter-caudate nucleus & 0.70 & 44.36 & $<0.05$ & Yes & 0.91/excellent \\
\hline White matter-putamen & 0.83 & 92.24 & $<0.05$ & Yes & 0.94/excellent \\
\hline White matter-globus pallidus & 0.82 & 84.43 & $<0.05$ & Yes & 0.98/excellent \\
\hline White matter-thalamus & 0.80 & 73.67 & $<0.05$ & Yes & 0.90/excellent \\
\hline White matter-internal capsule & 0.82 & 82.80 & $<0.05$ & Yes & 0.95/excellent \\
\hline Caudate nucleus-putamen & 0.32 & 8.82 & $<0.05$ & Yes & $0.88 /$ good \\
\hline Caudate nucleus-globus pallidus & 0.25 & 6.30 & $<0.05$ & Yes & $0.82 /$ good \\
\hline Caudate nucleus-thalamus & 0.42 & 13.67 & $<0.05$ & Yes & $0.90 /$ excellent \\
\hline Caudate nucleus-internal capsule & 0.78 & 66.07 & $<0.05$ & Yes & 0.98/excellent \\
\hline Putamen-globus pallidus & 0.32 & 8.87 & $<0.05$ & Yes & $0.85 /$ good \\
\hline Putamen-thalamus & 0.45 & 15.22 & $<0.05$ & Yes & $0.83 /$ good \\
\hline Putamen-internal capsule & 0.90 & 161.23 & $<0.05$ & Yes & 1/excellent \\
\hline Globus pallidus-thalamus & 0.59 & 26.67 & $<0.05$ & Yes & $0.89 /$ good \\
\hline Globus pallidus-internal capsule & 0.95 & 321.04 & $<0.05$ & Yes & 1/excellent \\
\hline Thalamus-internal capsule & 0.91 & 179.26 & $<0.05$ & Yes & 0.95/excellent \\
\hline \multicolumn{6}{|l|}{ Group 2} \\
\hline Cerebral crus-substantia nigra & 0.79 & 70.92 & $<0.05$ & Yes & 0.97/excellent \\
\hline Cerebral crus-red nucleus & 0.32 & 8.61 & $<0.05$ & Yes & $0.80 /$ good \\
\hline Cerebral crus-pons & 0.71 & 45.68 & $<0.05$ & Yes & $0.92 /$ excellent \\
\hline Cerebral crus-superior cerebellar peduncle & 0.69 & 41.79 & $<0.05$ & Yes & 0.91/excellent \\
\hline Cerebral crus-cerebellum & 0.9 & 162.81 & $<0.05$ & Yes & 0.94/excellent \\
\hline Substantia nigra-red nucleus & 0.71 & 45.83 & $<0.05$ & Yes & 0.90/excellent \\
\hline Substantia nigra-pons & 0.52 & 19.92 & $<0.05$ & Yes & $0.81 /$ good \\
\hline Substantia nigra-superior cerebellar peduncle & 0.61 & 29.18 & $<0.05$ & Yes & $0.88 / \operatorname{good}$ \\
\hline Substantia nigra-cerebellum & 0.6 & 28.17 & $<0.05$ & Yes & $0.92 /$ excellent \\
\hline Red nucleus-pons & 0.56 & 24.04 & $<0.05$ & Yes & $0.85 /$ good \\
\hline Red nucleus-superior cerebellar peduncle & 0.55 & 22.68 & $<0.05$ & Yes & $0.84 /$ good \\
\hline Red nucleus-cerebellum & 0.88 & 135.98 & $<0.05$ & Yes & 1/excellent \\
\hline Pons-superior cerebellar peduncle & 0.18 & 4.07 & $<0.05$ & Yes & $0.8 /$ good \\
\hline Pons-cerebellum & 0.77 & 60.98 & $<0.05$ & Yes & $0.90 /$ excellent \\
\hline Superior cerebellar peduncle-cerebellum & 0.76 & 57.61 & $<0.05$ & Yes & 0.93/excellent \\
\hline
\end{tabular}

The present study has noteworthy limitations:

Quantitative data were obtained from only 30 cases. In order to create a brain quantification database with regular quantitative T1, T2, and PD values, higher case numbers are required.
Quantitative measurements were conducted by one observer, which did not allow for analysis of reproducibility of measurements.

For the present study a $1.5 \mathrm{~T}$ machine was used. Especially since the $\mathrm{T} 1$ and $\mathrm{T} 2$ values have shown to be dependent on 
magnetic field strength, there is a need to assess the quantitative values for 3T applications as well [1, 3, 4]. Databases for further applications as previously described would have to contain quantitative $1.5 \mathrm{~T}$ and $3 \mathrm{~T}$ data. If the quantitative 1.5T and 3T values for one and the same tissue and organ are known, formulas for conversion of quantitative data generated with different magnetic field strength may be developed.

\section{Conclusions}

The assessed regular anatomical brain structures of cerebrum and brainstem/cerebellum can be characterized and differentiated based on a combination of their quantitative $\mathrm{T} 1, \mathrm{~T} 2$, and $\mathrm{PD}$ values. The data can be implemented in quantification databases and be corrected for different body temperatures using equations for temperature correction. This provides a base for future computer-aided diagnosis of brain pathologies and lesions in MR imaging.

Acknowledgments We would like to express our gratitude to our team of forensic pathologists and forensic technicians for their support with case handling.

\section{References}

1. Jackowski C, Warntjes MJ, Kihlberg J, Berge J, Thali MJ, Persson A (2011) Quantitative MRI in isotropic spatial resolution for forensic soft tissue documentation. Why and how? J Forensic Sci 56(1): $208-15$

2. Tashiro K, Shiotani S, Kobayashi T, Kaga K, Saito H, Someya S, Miyamoto K, Hayakawa H (2015) Cerebral relaxation times from postmortem MR imaging of adults. Magn Reson Med Sci 14(1): $51-6$

3. Zech WD, Schwendener N, Persson A, Warntjes MJ, Jackowski C (2015) Temperature dependence of postmortem MR quantification for soft tissue discrimination. Eur Radiol 25(8):2381-9

4. Zech WD, Schwendener N, Persson A, Warntjes MJ, Jackowski C (2015) Postmortem MR quantification of the heart for characterization and differentiation of ischaemic myocardial lesions. Eur Radiol 25(7):2067-73

5. Zech WD, Schwendener N, Persson A, Warntjes MJ, Riva F, Schuster F, Jackowski C (2015) Postmortem quantitative 1.5-T MRI for the differentiation and characterization of serous fluids, blood, CSF, and putrefied CSF. Int J Legal Med 129(5):1127-36

6. Warntjes JB, Dahlqvist O, Lundberg P (2007) Novel method for rapid, simultaneous $\mathrm{T} 1, \mathrm{~T} * 2$, and proton density quantification. Magn Reson Med 57:528-537

7. Warntjes JB, Leinhard OD, West J, Lundberg P (2008) Rapid magnetic resonance quantification on the brain: optimization for clinical usage. Magn Reson Med 60:320-329

8. Warntjes MJ, Kihlberg J, Engvall J (2010) Rapid T1 quantification based on 3D phase sensitive inversion recovery. BMC Med Imaging 10:19

9. Blystad I, Warntjes JB, Smedby O, Landtblom AM, Lundberg P, Larsson EM (2012) Synthetic MRI of the brain in a clinical setting. Acta Radiol 53:1158-1163
10. Doi K (2007) Computer-aided diagnosis in medical imaging: historical review, current status and future potential. Comput Med Imaging Graph 31(4-5):198-211

11. Shiraishi J, Li Q, Appelbaum D, Doi K (2011) Computer-aided diagnosis and artificial intelligence in clinical imaging. Semin Nucl Med 41(6):449-62

12. Birkl C, Langkammer C, Haybaeck J, Ernst C, Stollberger R, Fazekas F, Ropele S (2014) Temperature-induced changes of magnetic resonance relaxation times in the human brain: a postmortem study. Magn Reson Med 71(4):1575-80

13. Jonkman LE, Soriano AL, Amor S, Barkhof F, van der Valk P, Vrenken H, Geurts JJ (2015) Can MS lesion stages be distinguished with MRI? A postmortem MRI and histopathology study. J Neurol 262(4):1074-80

14. Egger C, Vaucher P, Doenz F, Palmiere C, Mangin P, Grabherr S (2012) Development and validation of a postmortem radiological alteration index: the RA-Index. Int J Legal Med 126(4):559-66

15. Synthetic MR products website. Available at: http://www. syntheticmr.com. Accessed September 30th, 2015.

16. Herrmann K, Johansen ML, Craig SE, Vincent J, Howell M, Gao Y, Lu L, Erokwu B, Agnes RS, Lu ZR, Pokorski JK, Basilion J, Gulani V, Griswold M, Flask C, Brady-Kalnay SM (2015) Molecular imaging of tumors using a quantitative T1 mapping technique via magnetic resonance imaging. Diagnostics (Basel) 5(3):318-332

17. Gracien RM, Reitz SC, Hof SM, Fleischer V, Zimmermann H, Droby A, Steinmetz H, Zipp F, Deichmann R, Klein JC (2015) Changes and variability of proton density and $\mathrm{T} 1$ relaxation times in early multiple sclerosis: MRI markers of neuronal damage in the cerebral cortex. Eur Radiol. 2015 Oct 22

18. Lundström C, Persson A, Ross S, Ljung P, Lindholm S, Gyllensvärd F, Ynnerman A (2012) State-of-the-art of visualization in post-mortem imaging. APMIS 120(4):316-326

19. Vågberg $M$, Lindqvist $T$, Ambarki $K$, Warntjes JB, Sundström $P$, Birgander R, Svenningsson A (2013) Automated determination of brain parenchymal fraction in multiple sclerosis. AJNR Am J Neuroradiol 34(3):498-504

20. Wu Y, Yang R, Jia S, Li Z, Zhou Z, Lou T (2014) Computer-aided diagnosis of early knee osteoarthritis based on MRI T2 mapping. Biomed Mater Eng 24(6):3379-88

21. Giannini V, Mazzetti S, Vignati A, Russo F, Bollito E, Porpiglia F, Stasi M, Regge D (2015) A fully automatic computer aided diagnosis system for peripheral zone prostate cancer detection using multi-parametric magnetic resonance imaging. Comput Med Imaging Graph

22. Gallego-Ortiz C, Martel AL (2015) Improving the accuracy of computer-aided diagnosis for breast MR imaging by differentiating between mass and nonmass lesions. Radiology

23. Ruder TD, Thali MJ, Hatch GM (2014) Essentials of forensic postmortem MR imaging in adults. Br J Radiol 87(1036):20130567

24. Schmidt TM, Fischer R, Acar S, Lorenzen M, Heinemann A, Wedegärtner U, Adam G, Yamamura J (2012) DWI of the brain: postmortal DWI of the brain in comparison with in vivo data. Forensic Sci Int 220(1-3):180-3

25. Yen K, Lövblad KO, Scheurer E, Ozdoba C, Thali MJ, Aghayev E, Jackowski C, Anon J, Frickey N, Zwygart K, Weis J, Dirnhofer R (2007) Post-mortem forensic neuroimaging: correlation of MSCT and MRI findings with autopsy results. Forensic Sci Int 173(1):2135

26. Flach PM, Schroth S, Schweitzer W, Ampanozi G, Slotboom J, Kiefer C, Germerott T, Thali MJ, El-Koussy M (2015) Deep into the fibers! Postmortem diffusion tensor imaging in forensic radiology. Am J Forensic Med Pathol 36(3):153-61

27. Scheurer E, Lovblad KO, Kreis R, Maier SE, Boesch C, Dirnhofer R, Yen K (2011) Forensic application of postmortem diffusionweighted and diffusion tensor MR imaging of the of the human brain in situ. AJNR Am J Neuroradiol 32(8):1518-24 
28. Añon J, Remonda L, Spreng A, Scheurer E, Schroth G, Boesch C, Thali M, Dirnhofer R, Yen K (2008) Traumatic extra-axial hemorrhage: correlation of postmortem MSCT, MRI, and forensic-pathological findings. J Magn Reson Imaging 28(4):823-36

29. Aghayev E, Yen K, Sonnenschein M, Ozdoba C, Thali M, Jackowski C, Dirnhofer R (2004) Virtopsy post-mortem multi-slice computed tomography (MSCT) and magnetic resonance imaging (MRI) demonstrating descending tonsillar herniation: comparison to clinical studies. Neuroradiology 46(7):559-64

30. Ruder TD, Hatch GM, Siegenthaler L, Ampanozi G, Mathier S, Thali MJ, Weber OM (2012) The influence of body temperature on image contrast in post mortem MRI. Eur J Radiol 81:1366-1370
31. Dickinson RJ, Hall AS, Hind AJ, Young IR (1986) Measurement of changes in tissue temperature using MR imaging. J Comput Assist Tomogr 10:468-472

32. Hausmann R, Vogel C, Seidl S, Betz P (2006) Value of morphological parameters for grading of brain swelling. Int J Legal Med 120(4):219-25

33. Yates A, Thelmo W, Pappius HM (1975) Postmortem changes in the chemistry and histology of normal and edematous brains. Am J Pathol 79(3):555-564

34. Dettmeyer RB (2011) Forensic neuropathology. In: Dettmeyer RB (ed) Forensic histopathology. Springer, Berlin, pp 413-438

35. Oehmichen M, Auer RN, Koenig HG (2009) Cell and tissue reactions. In: Forensic neuropathology and associated neurology. Springer, Berlin, pp 42-76 